\title{
Continuity of Genetic Risk for Aggressive Behavior Across the Life-Course
}

\author{
Camiel M. van der Laan ${ }^{1,2}(1)$. José J. Morosoli-García ${ }^{3} \cdot$ Steve G. A. van de Weijer ${ }^{2} \cdot$ Lucía Colodro-Conde $^{3}$ on \\ behalf of the ACTION Consortium $\cdot$ Michelle K. Lupton ${ }^{3} \cdot$ Brittany L. Mitchell ${ }^{3} \cdot$ Kerrie McAloney ${ }^{3} \cdot$ Richard Parker $^{3}$. \\ Jane M. Burns ${ }^{4} \cdot$ Ian B. Hickie ${ }^{5} \cdot$ René Pool $^{1}$. Jouke-Jan Hottenga ${ }^{1} \cdot$ Nicholas G. Martin $^{3} \cdot$ Sarah E. Medland $^{3}$. \\ Michel G. Nivard ${ }^{1} \cdot$ Dorret I. Boomsma ${ }^{1}$
}

Received: 31 January 2021 / Accepted: 23 June 2021 / Published online: 14 August 2021

(c) The Author(s) 2021

\begin{abstract}
We test whether genetic influences that explain individual differences in aggression in early life also explain individual differences across the life-course. In two cohorts from The Netherlands $(N=13,471)$ and Australia $(N=5628)$, polygenic scores (PGSs) were computed based on a genome-wide meta-analysis of childhood/adolescence aggression. In a novel analytic approach, we ran a mixed effects model for each age (Netherlands: 12-70 years, Australia: 16-73 years), with observations at the focus age weighted as 1 , and decaying weights for ages further away. We call this approach a 'rolling weights' model. In The Netherlands, the estimated effect of the PGS was relatively similar from age 12 to age 41, and decreased from age 41-70. In Australia, there was a peak in the effect of the PGS around age 40 years. These results are a first indication from a molecular genetics perspective that genetic influences on aggressive behavior that are expressed in childhood continue to play a role later in life.
\end{abstract}

Keywords Aggressive behavior $\cdot$ Aggression $\cdot$ Life-course $\cdot$ Development $\cdot$ Polygenic score $\cdot$ Rolling weights

\section{Introduction}

Aggression is broadly defined as common human behavior that intends to cause harm, by verbal, psychological, and physical means, to others (Baron and Richardson 1994; Anderson and Bushman 2002). Physical aggression tends to

Collaborators of the ACTION Consortium are listed in "Acknowledgement".

Camiel M. van der Laan

C.M.vander.Laan@vu.nl

1 Biological Psychology, Vrije Universiteit, Van der Boechorststraat 7, 1081 BT Amsterdam, The Netherlands

2 The Netherlands Institute for the Study of Crime and Law Enforcement, Amsterdam, The Netherlands

3 QIMR Berghofer Medical Research Institute, Brisbane, QLD, Australia

4 Faculty of Health Sciences, The University of Sydney, Sydney, Australia

5 Brain and Mind Centre, University of Sydney, Camperdown, Australia peak at age 2-4 years and then decreases (Alink et al. 2006; Cairns et al. 1989; Cairns and Cairns 1994; Karriker-Jaffe et al. 2008; Loeber and Stouthamer-Loeber 1998; Tremblay et al 2004; Tremblay 2010), as neurological, cognitive and social development empower children with other means to get what they want. Social or relational aggression emerges in the preschool years, continues through childhood and adolescence and subsequently declines in adulthood (e.g. Underwood 2003).

The relative positions in terms of aggression (i.e. rank order) in the population persist across the life-course (Pulkkinen and Pitkänen 1993; Tuvblad and Baker 2011). In other words, the most aggressive child often grows up to be the most aggressive adult (Farrington 1989). There has been some debate about the continuation of individual differences in aggression from childhood to adulthood. Moffitt (1993) argued that this statistical continuation is driven by a small number of highly aggressive individuals in a population who remain aggressive throughout their lives, the 'life-course persistent' individuals. The rest, she argues, are the 'adolescent limited' type, for whom aggressive behavior is limited to adolescence. Although it is clear that the 'life-course 
persistent' individuals explain part of the stability in aggression, Huesmann et al. (2009) showed that most individuals retain their relative position in a population, regardless of their starting position. Several factors have been identified that help explain individual differences in continuity of aggression, such as parenting, peers, socioeconomic and cultural context, mental processes and genetic predisposition (Boomsma 2015; Farrington 1989; Labella and Masten 2018; Murray and Farrington 2010; Tolan et al. 2013; Vuoksimaa et al. 2021).

Twin and family studies, mostly focusing on children, indicate that genetic factors explain around $50 \%$ of the variation in aggression (Veroude et al. 2016). Across the lifespan, heritability estimates of aggression and antisocial behavior seem to increase somewhat from childhood through adulthood, as the importance of shared environmental effects decreases (Tuvblad and Baker 2011; Waltes et al. 2016; Odintsova et al. 2019). Although individuals retain their genetic make-up throughout their lives, this does not necessarily imply that the same genetic variants play a role in aggression across the life-course. Studies with longitudinal twin designs show that genetic factors contribute significantly to the stability of aggression during preschool age, school age, and puberty (van Bijsterveldt et al. 2003; Porsch et al. 2016). These results led us to test the hypothesis that genetic variants that are expressed on aggression during childhood and adolescence also are significantly associated with aggression later in the life-course.

Odintsova and colleagues (2019) published an extensive overview of the current state of genomics aggression research, concluding that clear genome wide significant effects have not yet been found in genetic association studies (GWAS). This is partly attributable to the fact that aggression, like many other complex human behaviors, is influenced by a multitude of individual genetic variants, each of which likely has a small effect. From this 'polygenic' genetic architecture, arises the need for very large GWAS sample sizes. Ip and colleagues (2021) conducted a genome wide meta-analysis (GWAMA) of aggression phenotypes in children and adolescents, aged 3 to 18 years. In a GWAMA, results from GWAS in multiple cohorts are combined with the aim to increase statistical power to find associations between a genetic marker (usually a single nucleotide polymorphism, i.e. SNP) and an outcome (phenotype). If phenotypes and genetic effects are comparable across cohorts from different ages and backgrounds, small effects of SNPs that do not attain significance in a single cohort may be genome wide significant in the GWAMA. In the Ip et al. paper (2021), a total of 29 cohorts contributed 163 univariate GWAS to the early life aggression GWAMA. This resulted in a total of 328,935 observations from 87,485 unique individuals, aged 3 to 18 years. Observations were across multiple raters, coming from teachers, parents, and self-reports. The Ip et al. (2021) aggression GWAMA is the largest childhood aggression GWAS to date, but no single genome-wide significant hits were observed. Despite this lack of single significant hits, Ip et al. (2021) demonstrated that polygenic scores (PGSs), which sum the effects of a range of genetic markers, with markers included based on whether their $p$-value from the GWAS clears any of 16 thresholds between $\mathrm{P}=1$ and $\mathrm{P}<1.0 \mathrm{E}-5$, explained between 0.036 and $0.44 \%$ of the phenotypic variance in aggression in a hold-out sample of 7 year-old Dutch children $(N=4491)$. In an Australian hold-out sample, childhood PGSs explained up to $0.2 \%$ of retrospectively assessed childhood conduct disorder. PGSs performed best when markers where included with relatively lenient P-value thresholds, indicating the polygenic nature of aggression phenotypes. Although effect sizes were small, we expect the effect to be large enough to test the hypothesis that there are continuing genetic effects across the life-course.

\section{The Current Study}

In this study, we test the hypothesis that genetic risk factors, measured as DNA variants associated with increased aggressive behavior in early life (Ip et al. 2021), increase the risk of aggression across the life-course. We quantified the contribution of a large number of variants by computing PGSs, and tested their association with aggression in two cohorts from two different countries, namely The Netherlands and Australia. We introduce a novel method to assess differences in genetic influences across the life-course. In this approach we assess the effect of a PGS on aggression at ages 12-70 in The Netherlands, and 16-73 in Australia, by specifying 'rolling weights' for age. Within the framework of a linear mixed model, we model the effect of the PGS at each age represented in the data. At each age, we include phenotype information from surrounding ages. The phenotype information is weighted, where weights are centered at the focus age and decay further away from that center. With this method, sample size differences between ages are small, because more information than just the focus age is taken into account. Thereby, we mitigate the risk that sample size differences between ages drive the effects we find. If, for example, there are only few observations at age 25 , we can still use information on adjacent ages to imply the effects at age 25. A significant contribution in adults, of PGSs that are based on a discovery study in children and adolescents, would suggest a partially heritable origin of the stability of individual differences in aggression. 
Table 1 The Netherlands Twin Register

\begin{tabular}{lcl}
\hline Year & Observations & Mean age (SD) \\
\hline 1991 & 3325 & $17.95(2.24)$ \\
1995 & 3342 & $19.98(3.10)$ \\
1997 & 4714 & $26.73(10.46)$ \\
2000 & 6684 & $30.48(10.75)$ \\
2009 & 14,798 & $41.44(15.40)$ \\
2014 & 16,092 & $40.16(14.61)$ \\
$2005-2014$ (age twins: 14) & 11,080 & $15.35(1.54)$ \\
$2005-2014$ (age twins: 16) & 8075 & $17.43(1.60)$ \\
2005-2008 (age twins: 18) & 1516 & $18.88(1.94)$ \\
\hline
\end{tabular}

Collection of aggression data in adolescent and adult twins, their sibs, spouses and parents (1991-2014) and in young twins and their siblings (2005-2014)

This table includes all phenotype observations that were included to calculate IRT aggression scores

\section{Method}

\section{Participants}

Dutch participants are registered with The Netherlands Twin Register (NTR; Ligthart et al. 2019). Phenotype data on aggression were collected by survey in six of the data collection waves (1991, 1995, 1997, 2000, 2009, 2014) for twin families who registered as part of the Adult Netherlands Twin Register (ANTR). Twins, whose parents registered them as newborns as part of the Young Netherlands Twin Register (YNTR), and their siblings provided self-ratings of aggression at various ages of the twins. The total phenotyped sample in which aggression scores were computed (Table 1), consisted of families with twins, their siblings, spouses, and parents. In the final analyses only the genotyped individuals could be included. This genotyped sample included 29,454 measures from 13,471 genotyped individuals (62\% female) aged 12-70 years (Table 3, Fig. 1). In total, 8705 of these individuals completed more than one questionnaire. All genotyped individuals were from European ancestry, identified based on the top ten 1000-Genomes PCs (Abdellaoui et al. 2013) (Table 2).

Australian data came from studies on health and wellbeing collected at QIMR Berghofer Medical Research Institute (QIMRB). A total of 5628 genotyped participants from 2983 families from the Brisbane Longitudinal Twin Study (Wright and Martin 2004), Young and Well (16UP study, Mitchell et al. 2020) and Twenty Five and Up (25UP study, Mitchell et al. 2020) Genetics and Human Agency study (GHA, Morosoli 2020), and Prospective Imaging Study of Ageing (PISA; Lupton et al. 2021) completed surveys which included the Buss-Perry aggression questionnaire (Tables 3 and 4); 1603 people completed the questionnaire twice.
Table 2 The Netherlands Twin Register: characteristics of genotyped/ phenotyped sample

\begin{tabular}{lrllll}
\hline & $N$ subjects & $N$ measures & Mean age $(S D)$ & \multicolumn{2}{l}{$\begin{array}{l}\text { IRT aggres- } \\
\text { sion }\end{array}$} \\
\cline { 5 - 6 } & & & & Mean & $S D$ \\
\hline Total & 13,471 & 29,454 & $31.35(15.33)$ & -0.02 & 0.85 \\
Male & 5062 & 10,426 & $31.34(16.07)$ & -0.07 & 0.84 \\
Female & 8409 & 19,028 & $31.35(14.91)$ & 0.01 & 0.85 \\
\hline
\end{tabular}

IRT aggression item response theory aggression score, $S D$ standard deviation

Table 3 Australia

\begin{tabular}{lll}
\hline Study/year & Observations & Mean age (SD) \\
\hline 16UP (2014-2018) & 402 & $16.34(0.64)$ \\
25UP (2015-2019) & 2052 & $30.05(4.31)$ \\
GHA (2018-2020) & 2315 & $40.45(14.85)$ \\
PISA (2016-2020) & 2462 & $59.98(6.85)$ \\
\hline
\end{tabular}

Data collection (genotyped individuals)

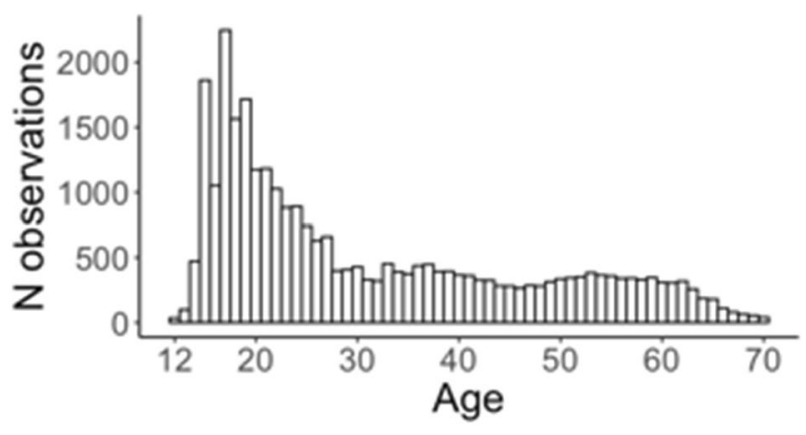

Fig. 1 The Netherlands. Age distribution

Table 4 Australia

\begin{tabular}{llllll}
\hline & $N$ subjects & $N$ measures & Mean age (SD) & \multicolumn{2}{l}{$\begin{array}{l}\text { IRT aggres- } \\
\text { sion }\end{array}$} \\
\cline { 5 - 6 } & & & & Mean & $S D$ \\
\hline Total & 5628 & 7231 & $42.81(16.71)$ & -0.01 & 0.95 \\
Male & 1904 & 2385 & $41.75(16.84)$ & 0.18 & 0.90 \\
Female & 3724 & 4846 & $43.33(16.62)$ & -0.11 & 0.96 \\
\hline
\end{tabular}

Sample characteristics genotyped individuals

IRT aggression item response theory aggression score, $S D$ standard deviation

At the time of completion, participants were aged 16-73 (Fig. 2). 


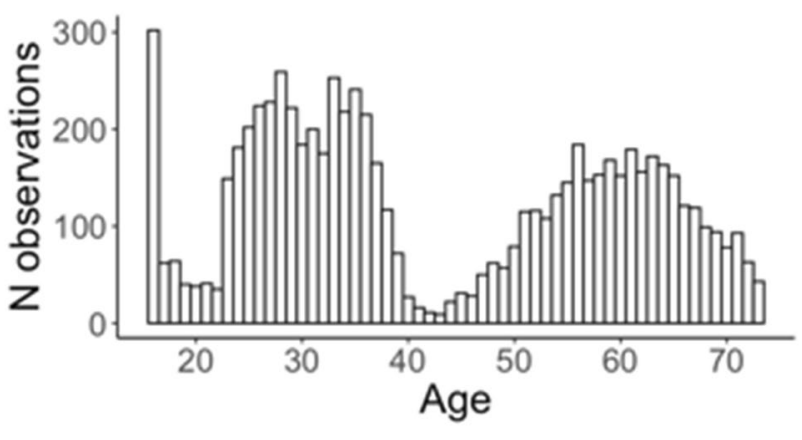

Fig. 2 Australia. Age distribution

\section{Phenotyping}

\section{The Netherlands}

All participants completed Achenbach System of Empirically Based Assessment self-report questionnaires (ASEBA; Achenbach et al. 2017), either the Youth Self-Report (YSR; Achenbach and Rescorla 2001) or the Adult Self-Report (ASR; Achenbach and Rescorla 2003). In the earlier ANTR surveys, the Young Adult Self Report (YASR) was administered. Surveys in each relevant data collection wave included between 15 and 20 items from the ASEBA aggressive behavior subscale. All items were scored on a three-level scale: $0=$ never, $1=$ sometimes, $2=$ often. Aggression scores were defined separately for each wave of data collection for all NTR participants (i.e. regardless of genotyping status, see Table 1) by Item-Response Theory (IRT; Embretson and Reise 2000) and calculated with the Generalized Partial Credit Model (GPCM) in R (R Core Team 2017), with the mirt package (Chalmers 2012). GPCM is an Item Response Theory model, developed to analyze polytomous data. For each wave of data collection, all participants with a maximum of two missing individual items were included in the GPCM. An IRT-aggression score has benefits over a simple sum-score, because it appropriately weights the relative contributions of individual items to a scale with a more favorable distribution, and takes into account missing data. By fitting a separate model for each wave of data collection, aggression scores for each participant are relative to all other participants in that wave of data collection, thereby filtering out potential 'wave' or data collection effects. Because the IRT score for each individual is relative to all other participants in the same wave of data collection, the mean IRT score for each wave is zero. This is reflected in the mean IRT aggression scores at each age (Fig. 3). The final overall IRT aggression score has a mean of 0.00 , and ranges from -1.6 to 4.4 , with a standard deviation of 0.86 . Only genotyped participants were included for further analysis. The genotyped sample did not differ much from the total sample, with

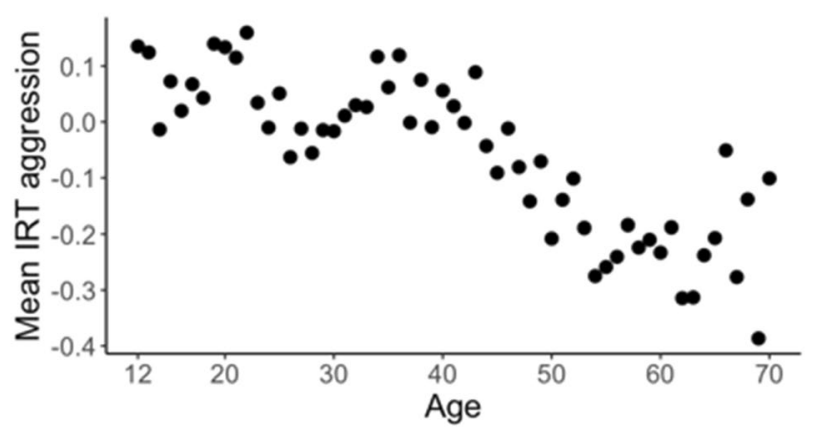

Fig. 3 Netherlands. Mean IRT aggression score for each age in the genotyped sample

a mean of -0.02 , range from -1.6 to 3.5 , and a standard deviation of 0.84 .

\section{Australia}

Aggressive behaviour was measured with the Buss-Perry Aggression Questionnaire. This is a 29-item questionnaire in which participants indicate the extent to which statements are characteristic of them (5-point Likert scale, from "extremely uncharacteristic of me" to "extremely characteristic of me", including some items that needed to be reversed). The questionnaire provides a total sum score and four subscores: physical aggression, Verbal aggression, Anger, and Hostility. For 107 out of 7231 observations, missing values on 1 to 6 individual items were imputed using multivariate imputation via the MICE R-package (van Buuren and Groothuis-Oudshoorn 2011). IRT aggression scores were calculated with mirt R-package (Chalmers 2012) within each study (i.e., 16UP, 25UP, GHA, and PISA) for the total aggression score and each of the subscales. Because the IRT score for each individual is relative to all other participants in the same study, the mean score for each study is zero. This is reflected in the mean aggression scores for each age (Fig. 4). The final overall IRT aggression score has

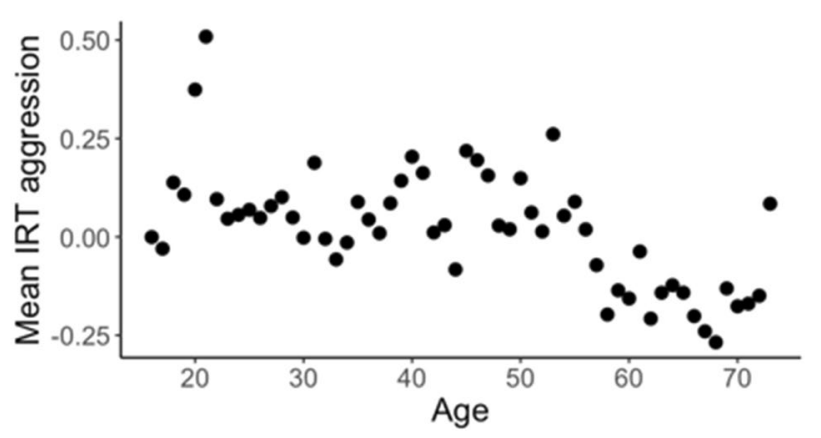

Fig. 4 Australia. Mean IRT aggression score for each age 
a mean of 0.0 , and ranges from -2.9 to 3.8 , with a standard deviation of 0.9 .

\section{Genotype Data}

The Netherlands: Participants were genotyped on multiple platforms: Affymetrix Axiom, Affymetrix 6.0, Illumina $1 \mathrm{M}$, Illumina 660, Illumina GSA, Perlegen Affymetrix. Samples with call rate $<0.90$, Plink heterozygosity $\mathrm{F}<-0.10$ or $\mathrm{F}>0.10$, and inconsistency of $\mathrm{X}$ chromosome genotypes with reported gender were excluded. SNPs with MAF $<1.0 \mathrm{E}-6$, HWE P-value $<1.0 \mathrm{E}-6$, and/or call rate $<0.95$ were removed. Genotype data were aligned with the 1000 Genomes reference panel, and filtered for SNPs with allele frequency differences from the CEU population larger than 0.20, palindromic SNPs, and DNA strand issues. DNA Identity By Descent (IBD) state was estimated for all individual pairs using Plink (Purcell et al. 2007) and King (Manichaikul et al. 2010) based on $\sim 10.8 \mathrm{k}$ SNPs that all platforms have in common. Samples were removed if IBD did not match expected family relations. CEU population outliers were removed from the data with Smartpca software, based on per platform 1000 Genomes PC projection. Per platform, data were phased using Eagle and imputed to 1000 Genomes with Minimac (Das et al. 2016). The final merged genotype data consist of 12,152,830 SNPs.

Australia: Genotyping was performed on DNA extracted from blood and saliva samples, on Illumina $317 \mathrm{~K}, 370 \mathrm{~K}$, $610 \mathrm{~K}$, ('1st generation'), GSA, or Core Exome plus Omnifamily ('2nd generation') arrays, and GenomeStudio software for genotype calling (Illumina Inc., 200 Lincoln Centre Dr, Foster City, CA 94404). This was followed by imputation from a common SNP set to the 1000 Genomes (Phase 3 Release 5) reference panel, a strategy that allows genotype data from different arrays to be combined. Samples with $<97.5 \%$ call rate, non-European ancestry ( $>6$ SD from the mean European-population cluster for PC1 and PC2) or with familial relationships incompatible with those reported by study participants were excluded. Observed markers were cleaned (by batch) for call rate $(\geq 95 \%)$; minor allele frequency ( $\geq 1 \%)$; Hardy-Weinberg equilibrium $\left(\mathrm{P} \geq 10^{-6}\right)$, GenCall score ( $\geq 0.15$ per genotype; mean $\geq 0.7)$ and standard Illumina filters, before integrating batches and re-running relationship and Mendelian checks. Phasing and imputation were carried out at the Michigan Imputation Server (https://imputationserver.sph.umich.edu/index.html\#!) using the 1000 Genomes Phase 3 Release 5 'mixed population' reference panel, with phasing by SHAPEIT followed by imputation using minimac 3 (Das et al. 2016), '1st generation' and '2nd generation' data were imputed separately due to poor overlap between typed markers. Imputation was based on 277,690 ('1st generation') and 240,297 ('2nd generation') typed markers (passing QC in all relevant batches); and the two were combined after imputation to maximise sample size, using for each individual the '1st generation' imputation if available, otherwise using the 'second generation' imputation. This resulted in 9,411,304 SNPs available for analysis, after quality control.

\section{Polygenic Score Construction}

We obtained effect sizes for the association between individual SNPs and aggression from the Ip et al. (2021) GWAMA after omitting the target samples, i.e. analyses were run with no participants from the Netherlands for the Dutch target sample, and no participants from Australia for the Australian target sample (GWAMA sample size for the Netherlands: $\mathrm{N}_{\mathrm{SNPs}}=7,722,825, \mathrm{~N}_{\text {measures }}=276,268, \mathrm{~N}_{\text {individuals }}=81,259$, $\mathrm{SNP}^{2} \mathrm{~h}^{2}=3.91 \%, \mathrm{SE}=0.42$. GWAMA sample size for Australia: $\mathrm{N}_{\mathrm{SNPs}}=7,762,065, \mathrm{~N}_{\text {measures }}=314,604$, $\left.\mathrm{N}_{\text {individuals }}=75,536, \mathrm{SNP}-\mathrm{h}^{2}=3.97 \%, \mathrm{SE}=0.46\right)$. We then computed PGSs for both cohorts with SBayesR V2.03 (Lloyd-Jones et al. 2019), using default settings.

\section{Statistical Analyses}

To ascertain the viability of predicting adult aggression with the PGS that is based on a discovery in 3- to 18-year-olds, we first model the association between the PGS and aggression in the total sample in the Netherlands and in Australia. Here, IRT aggression is predicted from the PGS, age, age ${ }^{2}$, sex, dummy variables for genotyping arrays, and five ancestrybased principal components. We control for the dependence between measures due to relatedness and repeated measures, by adding a random effect for families. Next, we model the effect of the PGS at specific ages. When investigating the effect of PGSs at specific ages, there is a risk that differences in sample size at each age may affect the results. To remedy this, we employ a novel weighted analytic approach in which we make use of more data when looking at specific ages. For each age for which data are available, ages 12 to 70 years in the Dutch context, and ages 16-73 years in the Australian context, we model aggression with the package lme4 (Bates et al. 2015), as a function of the PGS, age, age ${ }^{2}$, sex, dummy variables for genotyping arrays, and five ancestry-based principal components. This means a total of 59 analyses in The Netherlands and 58 in Australia. The models are fitted with weights that weight observations at the focus age as 1 and decay for ages further from that age. In this approach, sample size at each age includes ages around the focus age, but to a lesser extent. In other words, data on surrounding ages are included in the analyses, resulting in larger and more comparable sample sizes at different ages. At each age we control for the dependence between measures due to relatedness and repeated measures, by adding a random effect for families. This captures both dependence due to 
relatedness and dependence between longitudinal measures. Age covariates are included in the model because a range of ages is still present in each model, albeit with different weights. The mixed effects regression model at each age can be written as:

$Y_{m i f}=\beta_{0 f}+\beta_{\text {Agef }} * \mathrm{Age}_{m i}+\beta_{1-\mathrm{v}} * x_{m i, 1-13}+\varepsilon_{i f}$

In this notation, $\mathrm{Y}$ is the aggression outcome of individual $i$ in family $f$, the intercept $\beta_{0 f}$ is a combination of the population level intercept and the family-level deviation of that intercept, $\beta_{\text {Agef }}$ is the regression estimate for age and the family-level deviation of that estimate, $A g e_{m i}$ is the age of individual $i$ at measure $m, \beta_{1-\mathrm{v}}$ are the regression estimates for all the fixed effects (the PGS, age, age ${ }^{2}$, sex, dummy variables for genotyping arrays, and five ancestry-based principal components.), $x_{m i, 1-v}$ are the corresponding observed scores of individual $i$ at measure $m$ for each fixed effect, and $\epsilon_{i f}$ is the combined individual and group level error term. In matrix notation, this gives:

$Y=X \bullet \beta+Z \bullet b+\epsilon$

In this notation, $\mathrm{Y}$ is the matrix of observed responses, $\mathrm{X}$ and $\mathrm{Z}$ are the design matrices for fixed effects and mixed effects respectively, $\beta$ is the matrix of unknown fixed parameters, $b$ is the matrix of unknown random parameters, and $\epsilon$ is the vector of unobservable model errors. Because we apply weights to observations based on age, the least squares estimates for the fixed effects model parameters in this model are obtained by the following matrix formula, when we subtract away the random effects:

$\widehat{\beta}=\left[\begin{array}{c}\beta_{0} \\ \vdots \\ \beta_{v}\end{array}\right]=\left(X^{\prime} \cdot W \cdot X\right)^{-1} X^{\prime} \cdot W \cdot Y$

where $Y$ is the matrix of observed responses, $\beta_{0}$ to $\beta_{v}$ are the regression estimates for the intercept and all fixed effects, $X$ is the design matrix, and $W$ is the diagonal matrix of weights. The weights we apply to observations in the model are calculated in three steps. In step 1 the vector of ages is weighted as a function of center (age of interest) and shoulder (reflecting kurtosis in the distribution of weights):

$w_{1}=1-|c-x|^{s}$

In this notation, $w_{1}$ is the weights vector after step 1, $x$ is the vector of ages, $c$ is the center of the weights, and $s$ is the shoulder. In the second step, the weights vector from step 1 is scaled, by applying a min-max scalar:

$w_{2}=\left(w_{1}-\min \left(w_{1}\right)\right) /\left(\max \left(w_{1}\right)-\min \left(w_{1}\right)\right)$

In this notation, $w_{2}$ is the weights vector after step $2, w_{1}$ is the weights vector after step 1 . Third, the desired decay is applied to the scaled vector $w_{2}$, and the final diagonal matrix of weights is calculated:

$W=\operatorname{diag}\left(w_{2} e^{e^{k}}\right)$

where $W$ is the final diagonal matrix of weights, $w_{2}$ is the weights vector after step 2 and $e^{\mathrm{k}}$ represents the decay in the distribution of weights. In this approach, we opted for a shoulder of 1.5, and a decay of 25. See Fig. 5 for an example of the weights for ages 25 and 50. In general, a wider distribution of weights smooths the sample size and age-specific effects more, while a narrower distribution is more sensitive to fluctuations in sample size and age effects. We ran supplemental analyses to test the impact of wider and narrower age weight distributions (see Supplements Fig. 2 and 3).

All continuous fixed effects and the IRT aggression scores were standardized before the analyses. We employed bootstrapping to assess the robustness of the model estimated standard error. The approach was to sample complete families with replacement from the original data, 100 samples for each age-analysis. We found that model implied standard errors were slightly underestimated. Therefore, $95 \%$ confidence intervals reported in the results were calculated with the bootstrap standard errors. Significance is implied when the empirical (bootstrap) 95\% confidence intervals do not intersect zero.

\section{Results}

In the total genotyped Dutch sample $\left(\mathrm{N}_{\text {observations }}=29,454\right.$, $\mathrm{N}_{\text {individuals }}=13,471$ ), we first analyzed all data together without weighting the data. In this approach, the PGS was significantly associated with aggression, $\beta=0.05, S E=0.01$, pseudo $R^{2}=0.002, \mathrm{P}<0.001$. Next, we ran the age-specific age models with rolling weights. These analyses showed that the PGS was significantly related to IRT aggression from age 12 to age 41 ( $\beta=0.04-0.05$; Fig. 6 , Supplements Table I). After age 41, the effect of the PGS decreases, with confidence intervals that are close to, or below zero. The highest



Fig. 5 Example of weights with centers at ages 25 and 50 


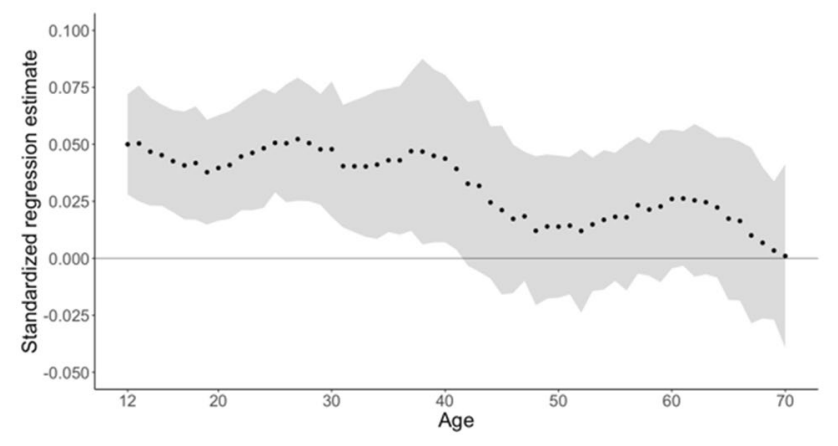

Fig. 6 Dutch data: standardized regression estimates for the effect of the PGS with bootstrapped 95\% confidence intervals (as grey banners)

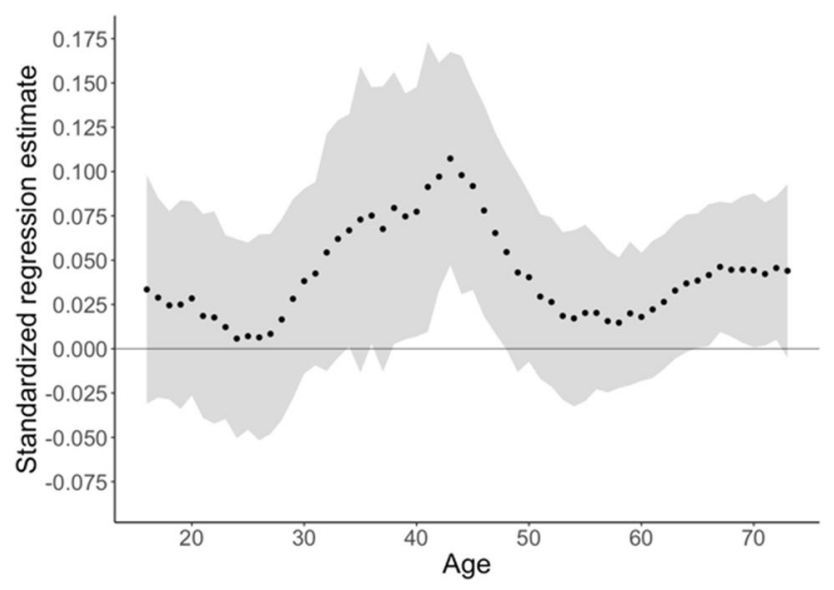

Fig. 7 Australian data: standardized regression estimates for the effect of the PGS with bootstrapped 95\% confidence intervals (as grey banners)

estimates for the effect of the PGSs are at around age 28. Supplemental analyses with wider and narrower age weights show that the width of the distribution affects the smoothing of the results, meaning that a narrower distribution of weights leads to more defined differences in PGS effects between ages (see Supplements Figs. 1 and 2).

In the total genotyped Australian sample without rolling weights $\left(\mathrm{N}_{\text {observations }}=7231, \mathrm{~N}_{\text {individuals }}=5,28\right)$, the PGS was significantly associated with adult and adolescent aggression, $\beta=0.04, S E=0.01$, pseudo $R^{2}=0.002, \mathrm{P}=0.002$. The specific age analyses from the model with rolling weights suggested a different association pattern across age (see Fig. 7, Supplements Table II) compared to the Dutch sample. The results did not indicate a downward trend in the regression estimates. Instead, the PGS was significantly related to aggression at ages 38 to $48(\beta=0.04-0.11)$.

In the Australian cohort we also investigated whether there are differences in prediction for the four subscales of the Buss Perry Aggression questionnaire (i.e. Physical aggression, Verbal aggression, Anger, and Hostility). The trends are very similar across all subscales. The peak around age 45 is clearly present for all subscales, albeit to a slightly lesser extent in verbal aggression. For figures of the regression estimates from the Buss Perry subscales see Supplements Fig. 3.

\section{Discussion}

In this study we introduce a new method to investigate the effect of a PGS across levels of a continuous moderating variable, in this case age. The approach is to run a linear model for each age in years present in the data. In each analysis, the phenotype information is weighted, with weights that are centered at the focus age and decay further away from that center. The strength of this method is that with each age analysis, information on proximal ages is taken into account, mitigating the risk that sample size differences at different ages will drive the effects we find. We applied this approach to assess the association between childhood aggression PGSs and aggression across the life-course, in two cohorts from The Netherlands and Australia.

In The Netherlands, the PGS was significantly related to aggression at ages 12 to 41 years. The effect of the PGS decreased from age 41-70 years. In the smaller Australian cohort, the effect of the PGS was significant at ages 38 to 48. Sample sizes at the peak of the PGS effect in Australia were between $N=193$ and $N=868$ (calculated as sum of weights, see Supplements Table II), which was relatively small. Because of these sample sizes, we should interpret this peak in effects with caution; for example, it could be driven by a small number of individuals that are not representative of the population. Effect sizes are small in both cohorts, with just under $0.2 \%$ explained variance in the full non-weighted models. We expect that effect sizes will increase as discovery GWAS sample sizes increase.

Although the effects are small, these results are the first indication from a molecular genetics perspective that genetic influences drive part of the continuity and stability of aggressive behavior, and that genetic effects in childhood persist across life, and thus across situations. This suggests that throughout people's lives, most notably in the Dutch context, developmental changes in individuals only slightly impact the polygenic effects on aggression that were apparent in childhood. These results correspond with findings from longitudinal twin studies on the stability of aggression in children (van Beijsterveldt et al. 2003; Eley et al. 2003; Porsch et al. 2016), and a twin study on the stability of externalizing psychopathology in adults (Gustavson et al. 2020), where genetic factors accounted for a large part of the stability over time. Van Beijsterveldt et al. (2003) also 
demonstrated that new genetic influences can contribute to stability of aggression across different ages. As such, the continuity of polygenic effects across the life-course covers only part of the genetic influences on the stability of aggression across the life-course.

A strength of this study is that we investigate the association between the PGS and aggression in two cohorts. This also implies, if we want to compare our results across cohorts, that there are several considerations, including differences in phenotyping, the impact of the discovery data, cultural differences between societies, and genetic differences between populations that may limit the feasibility of a reliable comparison. In the Dutch cohort, phenotyping was done with the ASEBA self-report questionnaires. In the Australian cohort, phenotyping was done with the Buss Perry Aggression self-report questionnaire. Differences in phenotyping are somewhat mitigated by using an IRT latent variable as dependent variable, instead of a sum score. Because different measurement instruments are used, this somewhat limits how we draw conclusions from the differences we found between the cohorts. A large percentage of participants in the discovery childhood aggression GWAMA from which we calculated our PGSs, were also scored with the ASEBA instruments, i.e. self-, parent- and teacher-reports (Ip et al. 2021), which relate directly to the ASEBA selfreport questionnaires used in the current Dutch sample. Correlations between self-, parent- and teacher-reports were not very high in Ip and colleagues' GWAMA. Still, we expect slightly greater power in the Dutch cohort compared to the Australian cohort, based on the similarities in the measurement instrument. Another potential source of dissimilarity is that the cohorts were not phenotyped in the same years. In The Netherlands, participants were phenotyped between 1991 and 2014, in Australia between 2014 and 2020, i.e. individuals that were phenotyped at the same age, are often not phenotyped in the same year. Van der Laan et al. (in press) show that self-reported aggression in The Netherlands declined from 1991 to 2015. Thus, differences between cohorts may be influenced by time effects. Another difference in phenotyping between the cohorts was that missing data $(<20 \%)$ were imputed prior to calculating IRT scores, while in the Dutch sample missing data $(<20 \%)$ were handled by the IRT models when calculating the scores.

The two discovery GWAMAs for the Dutch and Australian cohorts were also not identical. For The Netherlands, discovery data excluded all NTR participants. For Australia, discovery data excluded all Brisbane Longitudinal Twin Study and Prospective Imaging Study of Ageing participants. We opted for this strategy because leaving participants from both cohorts out of the discovery data would mean an unnecessary decrease in sample size. The differences in the discovery GWAMAs means that the PGSs in both samples are calculated based on overlapping, but not identical information. This may have resulted in slight differences in association between cohorts, although respectively only 5 and $17 \%$ of the Dutch and Australian discovery samples was not shared.

More generally, even if phenotyping is similar, cultural and genetic differences between populations can affect the magnitude, and thereby the merit, of PGS predictions. Cultural norms may influence aggression directly, and thereby moderate the effect of PGSs on aggression. One way to measure the effects of cultural differences on traits is by assessing generalizability of the measurement instruments via Confirmatory Factor Analysis (CFA), using the framework of measurement invariance (Millsap 2012). By assessing measurement invariance, we can test whether we measure the same underlying psychopathological trait when studying different societies. Because the Australian and Dutch cohorts did not phenotype by the same instruments, we cannot test measurement invariance directly in our sample. However, measurement invariance for the ASEBA self-report questionnaires is well documented. CFA of the eight-syndrome structure of the youth self-report, originally derived from a U.S. general population sample, plus clinically referred youths from Australia, England, and the United States (Achenbach and Rescorla 2001), fits YSR data from a wide range of societies (Ivanova et al. 2007). Fit indices were almost identical between Australia, RMSEA $=0.042$ and The Netherlands, RMSEA $=0.040$. Ivanova and colleagues (2015) also investigated generalizability of the eight-syndrome structure of the adult self-report (ASR) in 29 societies. Although The Netherlands and Australia were not included, model fit was good for all samples, with fit indices very similar to those found in the YSR CFAs. Generalizability of the Buss Perry aggression questionnaire has been less extensively studied, but validation is well documented for young Western adults (see for an overview Gerevich et al. 2007). Generalizability has been questioned for older and more diverse samples, but the four-factor structure (Physical aggression, Verbal Aggression, Hostility, and Anger) did replicate in a sample of Chilean students (Valdivia-Peralta et al. 2014), a slimmed down, translated 12 item version (Bryant and Smith 2001) replicated well in a sample of Hong Kong Chinese (Maxwell 2007), and all factors except Anger replicated in Hungarian adults with a mean age of 46.6 years (Gerevich et al. 2007).

Another source of differences between the population cohorts may be genetic heterogeneity. Based on genetic marker data, there are several ways to assess the comparability of samples from different populations. In an early comparison based on DNA tandem repeat polymorphisms from The Netherlands and Australia, Sullivan et al. (2006) estimated the fixation index $\left(\mathrm{F}_{\mathrm{ST}}\right)$ - the percentage genetic variability attributable to genetic differences between cohorts-to be only $0.3 \%$. The empirical variability between 
the Australian and Dutch cohorts was in fact smaller than for a combination of European samples $\left(\mathrm{F}_{\mathrm{ST}}=0.8 \%\right.$; Rosenberg 2021). More recently, Beck and colleagues (2019) studied interpopulation stratification between cohorts from The Netherlands and Australia, by analyzing genome wide SNP data. Negligible interpopulation stratification was confirmed by visualizing uncorrelated principal components and $\mathrm{F}_{\mathrm{ST}}$ estimations of $0.05 \%$. These findings should not be surprising, given the colonization history of Australia via immigrants from the UK, who are genetically very similar to Dutch individuals, as previously described with similarities in Y-chromosome haplogroups (Rosser et al. 2000).

At the moment, this genetic similarity between cohorts included in genomic studies is the rule rather than the exception. Around $78 \%$ of individuals included in GWAS are from European ancestry (EA; Buniello et al. 2019). The lack of individuals from non-EA populations included in GWAS, means that it is often unclear to what extent genetic effects generalize to diverse populations. Carlson and colleagues (2013) demonstrated that in non-EA populations, most GWAS-identified variants have allelic associations in the same direction as in EA populations, with none showing a statistically significant effect in the opposite direction. However, 25\% of tagSNPs had significantly different effect sizes in at least one non-EA population, most frequent in African Americans, with all differential effects diluted towards zero. Thus, associations between the PGS based on the Ip et al. (2021) discovery and aggression might be weaker in non-EA populations, as has been seen for other traits, such as obesity/BMI (Domingue et al. 2014; Belsky et al. 2013; Ware et al. 2017), height (Ware et al. 2017), educational attainment (Domingue et al. 2015; Ware et al. 2017; Lee et al. 2018), schizophrenia (Vilhalmsson et al. 2015; Ware et al. 2017; Vassos et al. 2017), and breast cancer (Ho et al. 2020). This means that advances through GWAS and PGS studies tended to benefit EA populations more than non-EA populations, especially when predicting health outcomes (Martin et al. 2019). The overrepresentation of EA participants in GWAS is partly because admixed populations were long considered inconvenient in gene discovery studies, as this led to population stratification issues. However, due to advances in GWAS methods, populations with mixed genetic backgrounds can now be included in GWAS to obtain accurate estimates of SNP effects, boost power, and improve fine-mapping of effects by leveraging linkage disequilibrium differences (Asimit et al. 2016; Atkinson et al. 2020). Beside the general benefits to gene discovery studies, the inclusion of diverse genetic backgrounds will improve our understanding of genetic liability across diverse populations, as demonstrated for example in a study of glycemic traits (Chen et al. 2021), where $30 \%$ of the participants were of non-European ancestry.
In summary, we investigated the continuity of polygenic effects on aggression across the life-course, in two cohorts from The Netherlands and Australia, with a novel weighted mixed effects regression approach. Our results suggest that the same genetic factors that explain part of the individual differences in aggression in childhood, also explain individual differences in adolescents and adults. The new method we employed shows promise in modeling genetic effects across levels of a continuous moderating variable, in way that smooths any possible effects due to sample size differences between those levels. The possibilities of reliably comparing results between the Dutch and Australian cohorts were limited because of differences in phenotyping and GWAMA discovery samples. When studying genetic liability in different populations, there are two main considerations: cultural/environmental differences and genetic differences. If we are interested in studying differences in genetic effects between populations with different cultural norms and environments, the optimal design is to look at populations with similar genetic backgrounds. For this to work, phenotyping has to be standardized across populations. To better understand genetic effects in diverse genetic populations, we need gene discovery studies that include diverse populations, so that predictions in non-EA populations are not dependent on EA discovery samples.

Supplementary Information The online version contains supplementary material available at https://doi.org/10.1007/s10519-021-10076-6.

Acknowledgements We warmly thank the twins and their family members for their continued support, generosity of time and interest in our research. We acknowledge the assistance of research assistants Natalie Garden and Reshika Chand and the support staff of The Netherlands Twin Registry and Genetic Epidemiology Laboratory at QIMR Berghofer Medical Research Institute. We also acknowledge the work done by each of the individual collaborators of the ACTION consortium: Hill F. Ip (Department of Biological Psychology, Vrije Universiteit Amsterdam, Amsterdam, The Netherlands), Camiel M. van der Laan (Department of Biological Psychology, Vrije Universiteit Amsterdam, Amsterdam, The Netherlands; Netherlands Institute for the Study of Crime and Law Enforcement, Amsterdam, The Netherlands), Eva M. L. Krapohl (Institute of Psychiatry, Psychology and Neuroscience, King's College London, London, UK), Isabell Brikell (Department of Medical Epidemiology and Biostatistics, Karolinska Institutet, Stockholm, Sweden), Cristina Sánchez-Mora (Department of Psychiatry, Hospital Universitari Vall d'Hebron, Barcelona, Spain; Biomedical Network Research Centre on Mental Health (CIBERSAM), Instituto de Salud Carlos III, Barcelona, Spain; Psychiatric Genetics Unit, Group of Psychiatry, Mental Health and Addiction, Vall d'Hebron Research Institute (VHIR), Universitat Autònoma de Barcelona, Barcelona, Spain), Ilja M. Nolte (Department of Epidemiology, University of Groningen, University Medical Center Groningen, Groningen, The Netherlands), Beate St Pourcain (MRC Integrative Epidemiology Unit, University of Bristol, Bristol, UK, Max Planck Institute for Psycholinguistics, Nijmegen, The Netherlands, Donders Institute for Brain, Cognition and Behaviour, Radboud University, Nijmegen, The Netherlands), Koen Bolhuis (Department of Child and Adolescent Psychiatry/ Psychology, Erasmus University Medical Center, Rotterdam, The Netherlands, Teemu Palviainen (Institute for Molecular Medicine 
FIMM, HiLife, University of Helsinki, Helsinki, Finland), Hadi Zafarmand (Department of Public Health, Amsterdam Public Health Research Institute, Amsterdam UMC, Academic Medical Center, University of Amsterdam, Amsterdam, The Netherlands; Department of Clinical Epidemiology, Biostatistics and Bioinformatics, Amsterdam Public Health Research Institute, Amsterdam UMC, Academic Medical Center, University of Amsterdam, Amsterdam, The Netherlands), Lucía Colodro-Conde (QIMR Berghofer Medical Research Institute, Brisbane, QLD, Australia), Scott Gordon (QIMR Berghofer Medical Research Institute, Brisbane, QLD, Australia), Tetyana Zayats (Department of Biomedicine, University of Bergen, Bergen, Norway, Analytic and Translational Genetics Unit, Department of Medicine, Massachusetts General Hospital and Harvard Medical School, Boston, MA, USA, Stanley Center for Psychiatric Research, Broad Institute of MIT and Harvard, Cambridge, MA, USA, Fazil Aliev ( (Department of Psychology, Virginia Commonwealth University, Richmond, VA, USA, Faculty of Business, Karabuk University, Karabuk, Turkey), Chang Jiang (Department of Epidemiology and Biostatistics, Michigan State University, East Lansing, MI, USA; Department of Biostatistics, University of Florida, Gainesville, FL, USA), Carol A. Wang (Faculty of Medicine and Health, School of Medicine and Public Health, The University of Newcastle, Callaghan, NSW, Australia), Gretchen Saunders (Department of Psychology, University of Minnesota, Minneapolis, MN, USA), Ville Karhunen (Department of Epidemiology and Biostatistics, MRC-PHE Centre for Environment and Health, School of Public Health, Imperial College London, London, UK), Anke R. Hammerschlag (Department of Biological Psychology, Vrije Universiteit Amsterdam, Amsterdam, The Netherlands; Child Health Research Centre, the University of Queensland, Brisbane, QLD, Australia; Amsterdam Public Health Research Institute, Amsterdam, The Netherlands), Daniel E. Adkins (Department of Sociology, College of Social and Behavioral Science, University of Utah, Salt Lake City, UT, USA; Department of Psychiatry, School of Medicine, University of Utah, Salt Lake City, UT, USA), Richard Border (Institute for Behavioral Genetics, University of Colorado Boulder, Boulder, CO, USA; Department of Psychology and Neuroscience, University of Colorado Boulder, Boulder, CO, USA; Department of Applied Mathematics, University of Colorado Boulder, Boulder, CO, USA), Roseann E. Peterson (Department of Psychiatry, Virginia Institute for Psychiatric and Behavioral Genetics, Virginia Commonwealth University, Richmond, VA, USA), Joseph A. Prinz (Center for Genomic and Computational Biology, Duke University, Durham, NC, USA), Elisabeth Thiering (Institute of Epidemiology, Helmholtz Zentrum München - German Research Center for Environmental Health, Neuherberg, Germany; Division of Metabolic Diseases and Nutritional Medicine, Dr. von Hauner Children's Hospital, Ludwig-Maximilians-University of Munich, Munich, Germany), Ilkka Seppälä (Department of Clinical Chemistry, Fimlab Laboratories, and Finnish Cardiovascular Research Center - Tampere, Faculty of Medicine and Health Technology, Tampere University, Tampere, Finland), Natàlia Vilor-Tejedor (ISGlobal, Barcelona Institute for Global Health, Barcelona, Spain; Universitat Pompeu Fabra (UPF), Barcelona, Spain; CIBER Epidemiología y Salud Pública (CIBERESP), Barcelona, Spain; Centre for Genomic Regulation (CRG), The Barcelona Institute of Science and Technology, Barcelona, Spain; Barcelona Beta Brain Research Center, Pasqual Maragall Foundation (FPM), Spain), Tarunveer S. Ahluwalia (COPSAC, Copenhagen Prospective Studies on Asthma in Childhood, Herlev and Gentofte Hospital, University of Copenhagen, Copenhagen, Denmark; Steno Diabetes Center Copenhagen, Gentofte, Denmark), Felix R. Day (MRC Epidemiology Unit, Institute of Metabolic Science, Cambridge Biomedical Campus, University of Cambridge School of Clinical Medicine, Cambridge, UK), Jouke-Jan Hottenga (Department of Biological Psychology, Vrije Universiteit Amsterdam, Amsterdam, The Netherlands), Andrea G. Allegrini (Institute of Psychiatry, Psychology and Neuroscience, King's College London, London), Kaili Rimfeld (Institute of Psychiatry, Psychology and Neuroscience, King's College
London, London), Qi Chen (Department of Medical Epidemiology and Biostatistics, Karolinska Institutet, Stockholm, Sweden), Yi Lu (Department of Medical Epidemiology and Biostatistics, Karolinska Institutet, Stockholm, Sweden), Joanna Martin (Department of Medical Epidemiology and Biostatistics, Karolinska Institutet, Stockholm, Sweden; MRC Centre for Neuropsychiatric Genetics and Genomics, Division of Psychological Medicine and Clinical Neurosciences, Cardiff University, Cardiff, UK), María Soler Artigas (Department of Psychiatry, Hospital Universitari Vall d'Hebron, Barcelona, Spain; Biomedical Network Research Centre on Mental Health (CIBERSAM), Instituto de Salud Carlos III, Barcelona, Spain; Psychiatric Genetics Unit, Group of Psychiatry, Mental Health and Addiction, Vall d'Hebron Research Institute (VHIR), Universitat Autònoma de Barcelona, Barcelona, Spain), Paula Rovira (Department of Psychiatry, Hospital Universitari Vall d'Hebron, Barcelona, Spain; Biomedical Network Research Centre on Mental Health (CIBERSAM), Instituto de Salud Carlos III, Barcelona, Spain; Psychiatric Genetics Unit, Group of Psychiatry, Mental Health and Addiction, Vall d'Hebron Research Institute (VHIR), Universitat Autònoma de Barcelona, Barcelona, Spain), Rosa Bosch (Department of Psychiatry, Hospital Universitari Vall d'Hebron, Barcelona, Spain; Biomedical Network Research Centre on Mental Health (CIBERSAM), Instituto de Salud Carlos III, Barcelona, Spain; Department of Psychiatry and Legal Medicine, Universitat Autònoma de Barcelona, Barcelona, Spain), Gemma Español (Department of Psychiatry, Hospital Universitari Vall d'Hebron, Barcelona, Spain), Josep Antoni Ramos Quiroga (Department of Psychiatry, Hospital Universitari Vall d'Hebron, Barcelona, Spain; Biomedical Network Research Centre on Mental Health (CIBERSAM), Instituto de Salud Carlos III, Barcelona, Spain; Psychiatric Genetics Unit, Group of Psychiatry, Mental Health and Addiction, Vall d'Hebron Research Institute (VHIR), Universitat Autònoma de Barcelona, Barcelona, Spain; Department of Psychiatry and Legal Medicine, Universitat Autònoma de Barcelona, Barcelona, Spain), Alexander Neumann (Department of Child and Adolescent Psychiatry/Psychology, Erasmus University Medical Center, Rotterdam, The Netherlands, Lady Davis Institute for Medical Research, Jewish General Hospital, Montreal, QC, Canada), Judith Ensink (Department of Child and Adolescent Psychiatry, Academic Medical Center, Amsterdam, The Netherlands; De Bascule, Academic Centre for Child and Adolescent Psychiatry, Amsterdam, The Netherlands), Katrina Grasby (QIMR Berghofer Medical Research Institute, Brisbane, QLD, Australia), José J. Morosoli (QIMR Berghofer Medical Research Institute, Brisbane, QLD, Australia), Xiaoran Tong (Department of Epidemiology and Biostatistics, Michigan State University, East Lansing, MI, USA, Department of Biostatistics, University of Florida, Gainesville, FL, USA), Shelby Marrington (School of Public Health, Faculty of Medicine, The University of Queensland, Herston, QLD, Australia), Christel Middeldorp (Department of Biological Psychology, Vrije Universiteit Amsterdam, Amsterdam, The Netherlands; Child Health Research Centre, the University of Queensland, Brisbane, QLD, Australia; Children's Health Queensland Hospital and Health Service, Child and Youth Mental Health Service, Brisbane, QLD, Australia), James G. Scott (School of Public Health, Faculty of Medicine, The University of Queensland, Herston, QLD, Australia; Metro North Mental Health, University of Queensland, St Lucia, QLD, Australia; Queensland Centre for Mental Health Research, St Lucia, QLD, Australia), Anna Vinkhuyzen (Institute for Molecular Bioscience, University of Queensland, St Lucia, QLD, Australia), Andrey A. Shabalin (Department of Psychiatry, School of Medicine, University of Utah, Salt Lake City, UT, USA), Robin Corley (Institute for Behavioral Genetics, University of Colorado Boulder, Boulder, CO, USA; Department of Ecology and Evolutionary Biology, University of Colorado Boulder, Boulder, CO, USA), Luke M. Evans (Institute for Behavioral Genetics, University of Colorado Boulder, Boulder, CO, USA; Department of Ecology and Evolutionary Biology, University of Colorado Boulder, Boulder, CO, USA), Karen Sugden (Center for Genomic and Computational Biology, Duke University, Durham, NC, USA; 
Department of Psychology and Neuroscience and Center for Genomic and Computational Biology, Duke University, Durham, NC, USA), Silvia Alemany (ISGlobal, Barcelona Institute for Global Health, Barcelona, Spain; Universitat Pompeu Fabra (UPF), Barcelona, Spain; CIBER Epidemiología y Salud Pública (CIBERESP), Barcelona, Spain), Lærke Sass (COPSAC, Copenhagen Prospective Studies on Asthma in Childhood, Herlev and Gentofte Hospital, University of Copenhagen, Copenhagen, Denmark), Rebecca Vinding (COPSAC, Copenhagen Prospective Studies on Asthma in Childhood, Herlev and Gentofte Hospital, University of Copenhagen, Copenhagen, Denmark), Kate Ruth (Genetics of Complex Traits, Royal Devon \& Exeter Hospital, University of Exeter Medical School, Exeter, UK), Jess Tyrrell (Genetics of Complex Traits, Royal Devon \& Exeter Hospital, University of Exeter Medical School, Exeter, UK), Gareth E. Davies (Avera Institute for Human Genetics, Sioux Falls, SD, USA), Erik A. Ehli (Avera Institute for Human Genetics, Sioux Falls, SD, USA), Fiona A. Hagenbeek (Department of Biological Psychology, Vrije Universiteit Amsterdam, Amsterdam, The Netherlands), Eveline De Zeeuw (Department of Biological Psychology, Vrije Universiteit Amsterdam, Amsterdam, The Netherlands), Toos C.E.M. Van Beijsterveldt (Department of Biological Psychology, Vrije Universiteit Amsterdam, Amsterdam, The Netherlands), Henrik Larsson (Department of Medical Epidemiology and Biostatistics, Karolinska Institutet, Stockholm, Sweden; School of Medical Sciences, Orebro University, Orebro, Sweden), Harold Snieder (Department of Epidemiology, University of Groningen, University Medical Center Groningen, Groningen, The Netherlands), Frank C. Verhulst (Department of Child and Adolescent Psychiatry/Psychology, Erasmus University Medical Center, Rotterdam, The Netherlands, Child and Adolescent Mental Health Centre, Mental Health Services Capital Region, Research Unit, Copenhagen University Hospital, Copenhagen, Denmark), Najaf Amin (Department of Epidemiology, Erasmus University Medical Center, Rotterdam, The Netherlands), Alyce M. Whipp (Institute for Molecular Medicine FIMM, HiLife, University of Helsinki, Helsinki, Finland), Tellervo Korhonen (Institute for Molecular Medicine FIMM, HiLife, University of Helsinki, Helsinki, Finland), Eero Vuoksimaa (Institute for Molecular Medicine FIMM, HiLife, University of Helsinki, Helsinki, Finland), Richard J. Rose (Department of Psychological and Brain Sciences, Indiana University, Bloomington, IN, USA), André G. Uitterlinden (Department of Epidemiology, Erasmus University Medical Center, Rotterdam, The Netherlands; Department of Internal Medicine, Erasmus University Medical Center, Rotterdam, The Netherlands; Netherlands Genomics Initiative (NGI)-sponsored Netherlands Consortium for Healthy Aging (NCHA), Leiden, The Netherlands), Andrew C. Heath (Washington University, St Louis, MO, USA), Pamela Madden (Washington University, St Louis, MO, USA), Jan Haavik (Department of Biomedicine, University of Bergen, Bergen, Norway, Division of Psychiatry, Haukeland University Hospital, Bergen, Norway), Jennifer R. Harris (Division of Health Data and Digitalisation, The Norwegian Institute of Public Health, Oslo, Norway), Øyvind Helgeland (Department of Genetics and Bioinformatics, Division of Health Data and Digitalization, The Norwegian Institute of Public Health, Bergen, Norway), Stefan Johansson (Department of Biomedicine, University of Bergen, Bergen, Norway; K.G. Jebsen Centre for Neuropsychiatric Disorders, Department of Clinical Science, University of Bergen, Bergen, Norway), Gun Peggy S. Knudsen (Division of Health Data and Digitalisation, The Norwegian Institute of Public Health, Oslo, Norway), Pal Rasmus Njolstad (Department of Clinical Science, University of Bergen, Bergen, Norway), Qing Lu (Department of Epidemiology and Biostatistics, Michigan State University, East Lansing, MI, USA, Department of Biostatistics, University of Florida, Gainesville, FL, USA), Alina Rodriguez (Department of Epidemiology and Biostatistics, MRC-PHE Centre for Environment and Health, School of Public Health, Imperial College London, London, UK; School of Psychology, University of Lincoln, Lincolnshire, UK), Anjali K. Henders (Institute for Molecular Bioscience, University of Queensland, St Lucia, QLD,
Australia), Abdullah Mamun (Institute for Social Science Research, University of Queensland, Long Pocket, QLD, Australia), Jackob M. Najman (School of Public Health, Faculty of Medicine, The University of Queensland, Herston, QLD, Australia), Sandy Brown (Department of Psychiatry, University of California, San Diego, CA, USA), Christian Hopfer (University of Colorado School of Medicine, Aurora, CO, USA), Kenneth Krauter (Department of Molecular, Cellular, and Developmental Biology, University of Colorado Boulder, Boulder, CO, USA), Chandra Reynolds (Department of Psychology, University of California Riverside, Riverside, CA, USA), Andrew Smolen (Institute for Behavioral Genetics, University of Colorado Boulder, Boulder, CO, USA), Michael Stallings (Institute for Behavioral Genetics, University of Colorado Boulder, Boulder, CO, USA; Department of Psychology and Neuroscience, University of Colorado Boulder, Boulder, CO, USA), Sally Wadsworth (Institute for Behavioral Genetics, University of Colorado Boulder, Boulder, CO, USA), Tamara L. Wall (Department of Psychiatry, University of California, San Diego, CA, USA), Judy L. Silberg (Department of Psychiatry, Virginia Institute for Psychiatric and Behavioral Genetics, Virginia Commonwealth University, Richmond, VA, USA; Department of Human \& Molecular Genetics, Virginia Institute for Psychiatric and Behavioral Genetics, Virginia Commonwealth University, Richmond, VA, USA), Allison Miller (Department of Pathology and Biomedical Science, and Carney Centre for Pharmacogenomics, University of Otago Christchurch, Christchurch Central City, New Zealand), Liisa Keltikangas-Järvinen (Department of Psychology and Logopedics, Faculty of Medicine, University of Helsinki, Helsinki, Finland), Christian Hakulinen (Department of Psychology and Logopedics, Faculty of Medicine, University of Helsinki, Helsinki, Finland), Laura Pulkki-Råback (Department of Psychology and Logopedics, Faculty of Medicine, University of Helsinki, Helsinki, Finland), Alexandra Havdahl (Nic Waals Institute, Lovisenberg Diaconal Hospital, Oslo, Norway; Department of Mental Disorders, Norwegian Institute of Public Health, Oslo, Norway), Per Magnus (Centre for Fertility and Health, Norwegian Institute of Public Health, Oslo, Norway), Olli T. Raitakari (Centre for Population Health Research, University of Turku and Turku University Hospital, Turku, Finland; Research Centre of Applied and Preventive Cardiovascular Medicine, University of Turku, Turku, Finland; Department of Clinical Physiology and Nuclear Medicine, Turku University Hospital, Turku, Finland), John R. B. Perry (MRC Epidemiology Unit, Institute of Metabolic Science, Cambridge Biomedical Campus, University of Cambridge School of Clinical Medicine, Cambridge, UK), Sabrina Llop (Epidemiology and Environmental Health Joint Research Unit, FISABIO-Universitat Jaume I-Universitat de València, Valencia, Spain; Spanish Consortium for Research on Epidemiology and Public Health (CIBERESP), Madrid, Spain), Maria-Jose Lopez-Espinosa (Epidemiology and Environmental Health Joint Research Unit, FISABIO-Universitat Jaume I-Universitat de València, Valencia, Spain; Spanish Consortium for Research on Epidemiology and Public Health (CIBERESP), Madrid, Spain; Faculty of Nursing and Chiropody, Universitat de València, Valencia, Spain), Klaus Bønnelykke (COPSAC, Copenhagen Prospective Studies on Asthma in Childhood, Herlev and Gentofte Hospital, University of Copenhagen, Copenhagen, Denmark), Hans Bisgaard (COPSAC, Copenhagen Prospective Studies on Asthma in Childhood, Herlev and Gentofte Hospital, University of Copenhagen, Copenhagen, Denmark), Jordi Sunyer (ISGlobal, Barcelona Institute for Global Health, Barcelona, Spain; Universitat Pompeu Fabra (UPF), Barcelona, Spain; CIBER Epidemiología y Salud Pública (CIBERESP), Barcelona, Spain; IMIM (Hospital del Mar Medical Research Institute), Barcelona, Spain), Terho Lehtimäki (Department of Clinical Chemistry, Fimlab Laboratories, and Finnish Cardiovascular Research Center - Tampere, Faculty of Medicine and Health Technology, Tampere University, Tampere, Finland), Louise Arseneault (Department of Psychiatry and Behavioral Sciences, Duke University School of Medicine, Durham, NC, USA), Marie Standl (Institute of Epidemiology, Helmholtz Zentrum München - German Research Center for 
Environmental Health, Neuherberg, Germany), Joachim Heinrich (Institute of Epidemiology, Helmholtz Zentrum München - German Research Center for Environmental Health, Neuherberg, Germany; Institute and Outpatient Clinic for Occupational, Social and Environmental Medicine, University of Munich Medical Center, LudwigMaximilians-Universität München, Munich, Germany; Allergy and Lung Health Unit, Melbourne School of Population and Global Health, University of Melbourne, Melbourne, VIC, Australia), Joseph Boden (Christchurch Health and Development Study, Department of Psychological Medicine, University of Otago Christchurch, Christchurch Central City, New Zealand), John Pearson (Biostatistics and Computational Biology Unit, Department of Pathology and Biomedical Science, University of Otago Christchurch, Christchurch Central City, New Zealand), L. John Horwood (Christchurch Health and Development Study, Department of Psychological Medicine, University of Otago Christchurch, Christchurch Central City, New Zealand), Martin Kennedy (Department of Pathology and Biomedical Science, and Carney Centre for Pharmacogenomics, University of Otago Christchurch, Christchurch Central City, New Zealand), Richie Poulton (Dunedin Multidisciplinary Health and Development Research Unit, University of Otago, Dunedin, New Zealand), Lindon J. Eaves (Department of Psychiatry, Virginia Institute for Psychiatric and Behavioral Genetics, Virginia Commonwealth University, Richmond, VA, USA; Department of Human \& Molecular Genetics, Virginia Institute for Psychiatric and Behavioral Genetics, Virginia Commonwealth University, Richmond, VA, USA), Hermine H. Maes (Department of Psychiatry, Virginia Institute for Psychiatric and Behavioral Genetics, Virginia Commonwealth University, Richmond, VA, USA; Department of Human \& Molecular Genetics, Virginia Institute for Psychiatric and Behavioral Genetics, Virginia Commonwealth University, Richmond, VA, USA; Massey Cancer Center, Virginia Commonwealth University, Richmond, VA, USA), John Hewitt (Institute for Behavioral Genetics, University of Colorado Boulder, Boulder, CO, USA; Department of Psychology and Neuroscience, University of Colorado Boulder, Boulder, CO, USA), William E. Copeland (Department of Psychiatry, College of Medicine, University of Vermont, Burlington, VT, USA), Elizabeth J. Costello (Department of Psychiatry, School of Medicine, Duke University, Durham, UK), Gail M. Williams (School of Public Health, Faculty of Medicine, The University of Queensland, Herston, QLD, Australia), Naomi Wray (Institute for Molecular Bioscience, University of Queensland, St Lucia, QLD, Australia; Queensland Brain Institute, Institute for Molecular Bioscience, University of Queensland, St Lucia, QLD, Australia), Marjo-Riitta Järvelin (Department of Epidemiology and Biostatistics, MRC-PHE Centre for Environment and Health, School of Public Health, Imperial College London, London, UK; Center for Life Course Health Research, Faculty of Medicine, University of Oulu, Oulu, Finland), Matt McGue (Department of Psychology, University of Minnesota, Minneapolis, MN, USA), William Iacono (Department of Psychology, University of Minnesota, Minneapolis, MN, USA), Avshalom Caspi (Center for Genomic and Computational Biology, Duke University, Durham, NC, USA; Department of Psychology and Neuroscience and Center for Genomic and Computational Biology, Duke University, Durham, NC, USA; Department of Psychiatry and Behavioral Sciences, Duke University School of Medicine, Durham, NC, USA; Social, Genetic, and Developmental Psychiatry Research Centre, Institute of Psychiatry, Psychology, and Neuroscience, King's College London, London, UK), Terrie E. Moffitt (Center for Genomic and Computational Biology, Duke University, Durham, NC, USA; Department of Psychology and Neuroscience and Center for Genomic and Computational Biology, Duke University, Durham, NC, USA; Department of Psychiatry and Behavioral Sciences, Duke University School of Medicine, Durham, NC, USA; Social, Genetic, and Developmental Psychiatry Research Centre, Institute of Psychiatry, Psychology, and Neuroscience, King's College London, London, UK), Andrew Whitehouse (Telethon Kids Institute, The University of Western Australia, Perth, WA, Australia), Craig E. Pennell (Faculty of
Medicine and Health, School of Medicine and Public Health, The University of Newcastle, Callaghan, NSW, Australia), Kelly L. Klump (Department of Psychology, Michigan State University, East Lansing, MI, USA), S. Alexandra Burt (Department of Psychology, Michigan State University, East Lansing, MI, USA), Danielle M. Dick (Department of Psychology, Virginia Commonwealth University, Richmond, VA, USA; Department of Human and Molecular Genetics, Virginia Commonwealth University, Richmond, VA, USA; College Behavioral and Emotional Health Institute, Virginia Commonwealth University, Richmond, VA, USA), Ted Reichborn-KjennerudDepartment of Mental Disorders, Norwegian Institute of Public Health, Oslo, Norway; Institute of Clinical Medicine, University of Oslo, Oslo, Norway), Nicholas G. Martin (QIMR Berghofer Medical Research Institute, Brisbane, QLD, Australia), Sarah E. Medland (QIMR Berghofer Medical Research Institute, Brisbane, QLD, Australia), Tanja Vrijkotte (Department of Public Health, Amsterdam Public Health Research Institute, Amsterdam UMC, Academic Medical Center, University of Amsterdam, Amsterdam, The Netherlands, Jaakko Kaprio (Institute for Molecular Medicine FIMM, HiLife, University of Helsinki, Helsinki, Finland; Department of Public Health, Medical Faculty, University of Helsinki, Helsinki, Finland), Henning Tiemeier (Department of Child and Adolescent Psychiatry/Psychology, Erasmus University Medical Center, Rotterdam, The Netherlands; Department of Social and Behavioral Science, Harvard TH Chan School of Public Health, Boston, MA, USA), George Davey Smith (MRC Integrative Epidemiology Unit, University of Bristol, Bristol, UK; Population Health Sciences, Bristol Medical School, University of Bristol, Bristol, UK), Catharina A. Hartman (Department of Psychiatry, University of Groningen, University Medical Center Groningen, Groningen, The Netherlands), Albertine J. Oldehinkel (Department of Psychiatry, University of Groningen, University Medical Center Groningen, Groningen, The Netherlands), Miquel Casas (Department of Psychiatry, Hospital Universitari Vall d'Hebron, Barcelona, Spain; Biomedical Network Research Centre on Mental Health (CIBERSAM), Instituto de Salud Carlos III, Barcelona, Spain; Psychiatric Genetics Unit, Group of Psychiatry, Mental Health and Addiction, Vall d'Hebron Research Institute (VHIR), Universitat Autònoma de Barcelona, Barcelona, Spain; Department of Psychiatry and Legal Medicine, Universitat Autònoma de Barcelona, Barcelona, Spain), Marta Ribasés (Department of Psychiatry, Hospital Universitari Vall d'Hebron, Barcelona, Spain; Biomedical Network Research Centre on Mental Health (CIBERSAM), Instituto de Salud Carlos III, Barcelona, Spain; 7, Paul Lichtenstein (Department of Medical Epidemiology and Biostatistics, Karolinska Institutet, Stockholm, Sweden), Sebastian Lundström (Gillberg Neuropsychiatry Centre, University of Gothenburg, Gothenburg, Sweden; Centre for Ethics, Law and Mental Health, University of Gothenburg, Gothenburg, Sweden), Robert Plomin (Institute of Psychiatry, Psychology and Neuroscience, King's College London, London), Meike Bartels (Department of Biological Psychology, Vrije Universiteit Amsterdam, Amsterdam, The Netherlands; Amsterdam Public Health Research Institute, Amsterdam, The Netherlands), Michel G. Nivard (Department of Biological Psychology, Vrije Universiteit Amsterdam, Amsterdam, The Netherlands; and Dorret I. Boomsma (Department of Biological Psychology, Vrije Universiteit Amsterdam, Amsterdam, The Netherlands; Amsterdam Public Health Research Institute, Amsterdam, The Netherlands).

Funding CvdL was supported by the Amsterdam Law and Behavior Institute (A-LAB; Vrije Universtiteit, Amsterdam). SvdW was suppported by NWO-grant 451-16-014. Data collection was made possible by multiple grants from The Netherlands Organization for Scientific Research (NWO): 575-25-006, 480-04-004, 904-61-090, 904-61-193, 400-05-717, 311-60008, SPI 56-464-14192 and the Avera Institute for Human Genetics. We gratefully acknowledge grant NWO 480-15001/674: Netherlands Twin Registry Repository: researching the interplay between genome and environment. MGN is supported by ZonMw 
grant: 'Genetics as a research tool: a natural experiment to elucidate the causal effects of social mobility on health' (pnr: 531003014) and ZonMw project: 'Can sex- and gender-specific gene expression and epigenetics explain sex-differences in disease prevalence and etiology?' (pnr: 849200011). Data collection in the Australian sample was made possible by multiple grants from NHMRC (APP1069141) and the John Templeton Foundation (Genetics and Human Agency Project). We also thank David Smyth and Scott Gordon for IT support and Richard Parker as project manager. The 16UP study was supported by the Young and Well Cooperative Research Centre, which was established and funded under the Australian Government's Cooperative Research Centres Program. The Prospective Imaging Study of Aging: Genes, Brain and Behaviour (PISA) is funded by a National Health and Medical Research Council (NHMRC) Boosting Dementia Research Initiative-Team Grant (APP1095227). Genome-wide SNP genotyping data for PISA participants is sourced from several genetic epidemiology studies led by NGM (with colleagues and collaborators) at QIMR Berghofer and elsewhere over the past 40 years. Principal sources of funding for these early studies were from grants to NGM from Australian NHMRC and to Andrew Heath and Pam Madden (Washington University, St Louis) from NIH (mainly NIAAA and NIDA) and we gratefully acknowledge these contributions. JJM was supported by a QIMR Berghofer International $\mathrm{PhD}$ Scholarship. LC-C was supported by a QIMR Berghofer Research Fellowship. SEM and NGM were funded by NHMRC investigator grants (APP1172917 and APP1172990).

Data Availability Available upon request.

Code Availability Please contact first author.

\section{Declarations}

Conflict of interest Camiel M. van der Laan, José J. Morosoli-García, Steve G. A. van de Weijer, Lucía Colodro-Conde, ACTION consortium, Michelle K. Lupton, Brittany L. Mitchell, Kerrie McAloney, Richard Parker, Jane M. Burns, Ian B. Hickie, René Pool, Jouke-Jan Hottenga, Nicholas G. Martin, Sarah E. Medland, Michel G. Nivard, and Dorret I. Boomsma declare that they have no conflict of interest.

Ethical approval This study was performed in accordance with the ethical standards as laid down in the 1964 Declaration of Helsinki. Ethics approval was obtained for each separate data source: The Netherlands Twin Register (NTR; Ligthart et al. 2019), the Brisbane Longitudinal Twin Study (Wright and Martin 2004)—Young and Well (16UP study; Kirk et al., in press) and Twenty Five and Up (25UP study; Mitchell et al. 2020) Genetics and Human Agency study (GHA; Morosoli 2020), and Prospective Imaging Study of Ageing (PISA; Lupton et al. 2021).

Consent to Participate Informed consent was obtained from all participants.

Consent for Publication Not applicable.

Open Access This article is licensed under a Creative Commons Attribution 4.0 International License, which permits use, sharing, adaptation, distribution and reproduction in any medium or format, as long as you give appropriate credit to the original author(s) and the source, provide a link to the Creative Commons licence, and indicate if changes were made. The images or other third party material in this article are included in the article's Creative Commons licence, unless indicated otherwise in a credit line to the material. If material is not included in the article's Creative Commons licence and your intended use is not permitted by statutory regulation or exceeds the permitted use, you will need to obtain permission directly from the copyright holder. To view a copy of this licence, visit http://creativecommons.org/licenses/by/4.0/.

\section{References}

Abdellaoui A, Hottenga JJ, De Knijff P, Nivard MG, Xiao X, Scheet P, et al (2013) Population structure, migration, and diversifying selection in The Netherlands. Eur J Hum Genet 21(11):1277-1285

Achenbach TM, Rescorla LA (2001) Manual for the ASEBA schoolage forms \& profiles. University of Vermont, Burlington

Achenbach TM, Rescorla LA (2003) Manual for the ASEBA adult forms \& profiles. University of Vermont, Burlington

Achenbach TM, Ivanova MY, Rescorla LA (2017) Empirically based assessment and taxonomy of psychopathology for ages 11/2-90+ years: developmental, multi-informant, and multicultural findings. Compr Psychiatry 9:4-18

Alink LR, Mesman J, Van Zeij1 J, Stolk M, Juffer F, Koot H, et al (2006) The early childhood aggression curve: development of physical aggression in 10- to 50-month-old children. Child Dev 77(4):954-966

Anderson CA, Bushman BJ (2002) Human aggression. Annu Rev Psychol 53:27-51

Asimit JL, Hatzikotoulas K, McCarthy M, Morris AP, Zeggini E (2016) Trans-ethnic study design approaches for fine-mapping. Eur J Hum Genet 24(9):1330-1336

Atkinson EG, Maihofer AX, Kanai M, Martin AR, Karczewski KJ, Santoro ML, et al. (2020) Tractor: a framework allowing for improved inclusion of admixed individuals in large-scale association studies. https://www.biorxiv.org/content/https://doi.org/ 10.1101/2020.05.17.100727v1

Baron RA, Richardson DR (1994) Human aggression, 2nd edn. Plenum, New York

Bates D, Maechler M, Bolker B, Walker S (2015) Fitting linear mixedeffects models using lme4. J Stat Softw 67(1):1-48

Beck J, Hottenga JJ, Mbarek H, Finnicum C, Ehli E, Hur Y, et al (2019) Genetic similarity assessment of twin-family populations by custom-designed genotyping array. Twin Res Hum Genet 22(4):210-219

Belsky DW, Moffitt TE, Sugden K, Williams B, Houts R, McCarthy J, Caspi A (2013) Development and evaluation of a genetic risk score for obesity. Biodemography Soc Biol 59(1):85-100

Boomsma DI (2015) Aggression in children: unravelling the interplay of genes and environment through (epi)genetics and metabolomics. J Ped Neo Ind Med 4:e040251

Boomsma DI, Vink JM, van Beijsterveldt CEM, de Geus EJC, Beem AL, Mulder EJCM, et al (2002) Netherlands twin register: a focus on longitudinal research. Twin Res 5:401-406

Boomsma DI, de Geus EJC, Vink JM, Stubbe JH, Distel MA, Hottenga JJ, Willemsen G (2006) Netherlands Twin Register: from twins to twin families. Twin Res Hum Genet 9:849-857

Buniello A, MacArthur J, Cerezo M, Harris LW, Hayhurst J, Malangone C, et al (2019) The NHGRI-EBI GWAS Catalog of published genome-wide association studies, targeted arrays and summary statistics 2019. Nucleic Acids Res 47:1005-1012

Buss AH, Perry M (1992) The aggression questionnaire. J Pers Soc Psychol 63(3):452

Cairns RB, Cairns BD (1994) Life lines and risks: pathways of youth in our time. Cambridge University Press, New York

Cairns RB, Cairns BD, Neckerman H, Ferguson L, Gariépy J (1989) Growth and aggression: I. Childhood to early adolescence. Dev Psychol 25(2):320-330

Carlson CS, Matise TC, North KE, Haiman CA, Fesinmeyer MD, Buyske $\mathrm{S}$ et al (2013) Generalization and dilution of association 
results from european gwas in populations of non-european ancestry: the page study. PLos Biol 11(9):e1001661

Chalmers RP (2012) mirt: a multidimensional item response theory package for the R environment. J Stat Softw 48(6):1-29

Chen J, Spracklen CN, Marenne G, Varshney A, Corbin LJ, Luan J et al (2021) The trans-ancestral genomic architecture of glycemic traits. Nat Genet 53(6):840-860

Das S, Forer L, Schönherr S, Sidore C, Locke AE, Vrieze SI, et al (2016) Next-generation genotype imputation service and methods. Nat Genet 48:1284-1287

Domingue BW, Belsky DW, Harris KM, Smolen A, McQueen MB, Boardman JD (2014) Polygenic risk predicts obesity in both white and black young adults. PLoS ONE 9(7):e101596

Domingue BW, Belsky D, Conley D, Harris KM, Boardman JD (2015) Polygenic influence on educational attainment: new evidence from The National Longitudinal Study of Adolescent to Adult Health. AERA Open 1(3):1-13

Eley TC, Lichtenstein P, Moffitt TE (2003) A longitudinal behavioral genetic analysis of the etiology of aggressive and nonaggressive antisocial behavior. Dev Psychopathol 15(2):383-402

Embretson SE, Reise SP (2000) Item response theory for psychologists. Lawrence Erlbaum, Mahwah

Farrington DP (1989) Early predictors of adolescent aggression and adult violence. Violence Vict 4:79-138

Gerevich J, Bácskai E, Czobor P (2007) The generalizability of the buss-perry aggression questionnaire. Int J Methods Psychiatr Res 16(3):124-136

Gustavson DE, Franz CE, Panizzon MS, Lyons MJ, Kremen WS (2020) Internalizing and externalizing psychopathology in middle age: genetic and environmental architecture and stability of symptoms over 15 to 20 years. Psychol Med 50(9):1530-1538

Ho W-K, Tan M-M, Mavaddat N, Tai M-C, Mariapun S, Li J, et al (2020) European polygenic risk score for prediction of breast cancer shows similar performance in asian women. Nat Commun 11(1):1-11

Huesmann LR, Dubow EF, Boxer P (2009) Continuity of aggression from childhood to early adulthood as a predictor of life outcomes: implications for the adolescent-limited and life-course-persistent models. Aggr Behav 35(2):136-149. https://doi.org/10.1002/ab. 20300

Ip HF, van der Laan CM, Brikell I, Sánchez-Mora C, Nolte IM, St. Pourcain B, et al (2021) Genetic association study of childhood aggression across raters, instruments, and age. Transl Psychiatry 11:413. https://doi.org/10.1038/s41398-021-01480-x

Ivanova MY, Achenbach TM, Rescorla LA, Dumenci L, Almqvist F, Bilenberg N, et al (2007) The generalizability of the youth selfreport syndrome structure in 23 societies. J Consult Clin Psychol 75(5):729-738

Ivanova MY, Achenbach TM, Rescorla LA, Turner LV, Ahmeti-Pronaj A, Au A, et al (2015) Syndromes of self-reported psychopathology for ages 18-59 in 29 societies. J Psychopathol Behav Assess 37(2):171-183

Karriker-Jaffe K, Foshee V, Ennett S, Suchindran C (2008) The development of aggression during adolescence: sex differences in trajectories of physical and social aggression among youth in rural areas. J Abnorm Child Psychol 36(8):1227-1236

Labella M, Masten A (2018) Family influences on the development of aggression and violence. Curr Opin Psychol 19:11-16

Lee JJ, Wedow R, Okbay A, Kong E, Maghzian O, Zacher M et al (2018) Gene discovery and polygenic prediction from a genomewide association study of educational attainment in 1.1 million individuals. Nat Genet 50(8):1112-1121

Ligthart L, van Beijsterveldt CEM, Kevenaar ST, de Zeeuw E, van Bergen E, Bruins S, et al (2019) The Netherlands Twin Register: longitudinal research based on twin research and twin-family designs. Twin Res Hum Genet 15:1-14
Lloyd-Jones LR, Zeng J, Sidorenko J, Yengo L, Moser G, Kemper KE, et al (2019) Improved polygenic prediction by Bayesian multiple regression on summary statistics. Nat Commun 10(1):1-11

Loeber R, Stouthamer-Loeber M (1998) Development of juvenile aggression and violence. Some common misconceptions and controversies. Am Psychol 53(2):242-259

Lupton MK, Robinson GA, Adam RJ, Rose S, Byrne GJ, Salvado O et al (2021) A prospective cohort study of prodromal Alzheimer's disease: prospective imaging study of ageing: genes, brain and behaviour (PISA). NeuroImage 29:102527

Manichaikul A, Mychaleckyj JC, Rich SS, Daly K, Sale M, Chen WM (2010) Robust relationship inference in genome-wide association studies. Bioinformatics (Oxford, England) 26(22):2867-2873. https://doi.org/10.1093/bioinformatics/btq559

Martin AR, Kanai M, Kamatani Y, Okada Y, Neale BM, Daly MJ (2019) Clinical use of current polygenic risk scores may exacerbate health disparities. Nat Genet 51(4):584-591

Maxwell JP (2007) Development and preliminary validation of a chinese version of the buss-perry aggression questionnaire in a population of hong kong chinese. J Pers Assess 88(3):284-294

Millsap R (2012) Statistical approaches to measurement invariance. Routlede, New York

Mitchell BL, Campos AI, Rentería ME, Parker R, et al (2019) Twentyfive and up (25Up) study: a new wave of the Brisbane Longitudinal Twin Study. Twin Res Hum Genet 22(3):154-163

Mitchell BL, Kirk KM, McAloney K, Wright MJ, Davenport TA, et al (2020) 16up: outline of a study investigating wellbeing and information and communication technology use in adolescent twins. Twin Res Hum Genet 23(6):345-357

Moffitt TE (1993) Adolescence-limited and life-course-persistent antisocial behavior: a developmental taxonomy. Psychol Rev 100:674-701

Morosoli JJ (2020) Overcoming genetic determinism: A psychological perspective on public understanding of human complex trait genetics. (Unpublished doctoral dissertation). University of Queensland, Brisbane, Australia.

Murray J, Farrington DP (2010) Risk factors for conduct disorder and delinquency: key findings from longitudinal studies. Can J Psychiatry 55(10):633-42

Odintsova VV, Roetman PJ, Ip HF, Pool R, Van der Laan CM, Tona K-D, et al (2019) Genomics of human aggression: current state of genome-wide studies and an automated systematic review tool. Psychiatr Genet 29(5):170-190

Porsch RM, Middeldorp CM, Cherny SS, Krapohl E, van Beijsterveldt CEM, Loukola A, et al (2016) Longitudinal heritability of childhood aggression. Am J Med Genet B Neuropsychiatr Genet 171(5):697-707

Pulkkinen L, Pitkänen T (1993) Contiuities in aggressive behavior from childhood to adulthood. Aggr Behav 19:249-264

Purcell S, Neale B, Todd-Brown K, Thomas L, Ferreira MAR, Bender D, et al (2007) PLINK: a toolset for whole-genome association and population-based linkage analysis. Am J Hum Genet 81(3):559-575

R Core Team (2017) R: a language and environment for statistical computing. R Foundation for Statistical Computing, Vienna

Rosenberg NA (2021) A population-genetic perspective on the similarities and differences among worldwide human populations. Hum Biol 92(3):135-152. https://doi.org/10.13110/humanbiolo gy.92.3.02

Rosenberg NA, Pritchard JK, Weber JL, Cann HM, Kidd KK, Zhivotovsky LA, Feldman MW (2002) Genetic structure of human populations. Science 298(5602):2381-2385

Rosser ZH, Zerjal T, Hurles ME, Adojaan M, Alavantic D, Amorim A, et al (2000) Y-chromosomal diversity in Europe is clinal and influenced primarily by geography, rather than by language. Am J Hum Genet 67(6):1526-1543 
Tolan PH, Dodge K, Rutter M (2013) Tracking the multiple pathways of parent and family influence on disruptive behavior disorders. In: Tolan PH, Leventhal BL (eds) Disruptive behavior disorders. New York, Springer, pp 161-191

Tremblay RE (2010) Developmental origins of disruptive behaviour problems: the 'original sin' hypothesis, epigenetics and their consequences for prevention. J Child Psychol Psychiatry 51(4):341-367

Tremblay RE, Nagin DS, Séguin JR, Zoccolillo M, Zelazo PD, Boivin M, et al (2004) Physical aggression during early childhood: trajectories and predictors. Pediatrics 114(1):43-50

Tuvblad C, Baker LA (2011) Human aggression across the lifespan: genetic propensities and environmental moderators. Adv Genet $75: 171-214$

Underwood MK (2003) Social aggression among girls. Guilford, New York

Valdivia-Peralta M, Fonseca-Pedrero E, González-Bravo L, LemosGiráldez S (2014) Psychometric properties of the AQ aggression scale in Chilean students. Psicothema 26(1):39-46

van Beijsterveldt CEM, Bartels M, Hudziak JJ, Boomsma DI (2003) Causes of stability of aggression from early childhood to adolescence: a longitudinal genetic analysis in Dutch twins. Behav Genet 33(5):591-605

van Buuren S, Groothuis-Oudshoorn K (2011) Mice: multivariate imputation by chained equations in R. J Stat Softw 45(3):1-67

van der Laan CM, van de Weijer SGA, Nivard MG, Boomsma DI (in press) Familial clustering of trends in aggression. J Quant Criminol

Vassos E, Di Forti M, Coleman J, Iyegbe C, Prata D, Euesden J, et al (2017) An examination of polygenic score risk prediction in individuals with first-episode psychosis. Biol Psychiat 81(6):470-477
Veroude K, Zhang-James Y, Fernàndez-Castillo N, Bakker MJ, Cormand B, Faraone SV (2016) Genetics of aggressive behavior: an overview. Am J Med Genet Part B Neuropsychiatr Genet 171:3-43

Vilhjálmsson BJ, Yang J, Finucane HK, Gusev A, Lindström S, Ripke $S$, et al (2015) Modeling linkage disequilibrium increases accuracy of polygenic risk scores. Am J Hum Genet 97(4):576-592

Vuoksimaa E, Rose RJ, Pulkkinen L, Palviainen T, Rimfeld K, Lundström S, et al (2021) Higher aggression is related to poorer academic performance in compulsory education. J Child Psychol Psychiatry 62(3):327-338

Waltes R, Chiocchetti AG, Freitag CM (2016) The neurobiological basis of human aggression: a review on genetic and epigenetic mechanisms. Am J Med Genet B Neuropsychiatr Genet 171(5):650-675. https://doi.org/10.1002/ajmg.b.32388

Ware, EB, Schmitz, LL, Faul, JD, Gard, A, Mitchell, C, Smith, JA, et al (2017) Heterogeneity in polygenic scores for common human traits (bioRxiv). https://doi.org/10.1101/106062

Wray NR, Lee SH, Mehta D, Vinkhuyzen AAE, Dudbridge F, Middeldorp CM (2014) Research review: polygenic methods and their application to psychiatric traits. J Child Psychol Psychiatry 5(10):1068-1087

Wright MJ, Martin NG (2004) Brisbane Adolescent Twin Study: outline of study methods and research projects. Aust J Psychol 56:65-78

Publisher's Note Springer Nature remains neutral with regard to jurisdictional claims in published maps and institutional affiliations. 